\title{
LOCAL PROJECTION STABILIZATION OF EQUAL ORDER INTERPOLATION APPLIED TO THE STOKES PROBLEM
}

\author{
SASHIKUMAAR GANESAN, GUNAR MATTHIES, AND LUTZ TOBISKA
}

\begin{abstract}
The local projection stabilization allows us to circumvent the Babuška-Brezzi condition and to use equal order interpolation for discretizing the Stokes problem. The projection is usually done in a two-level approach by projecting the pressure gradient onto a discontinuous finite element space living on a patch of elements. We propose a new local projection stabilization method based on (possibly) enriched finite element spaces and discontinuous projection spaces defined on the same mesh. Optimal order of convergence is shown for pairs of approximation and projection spaces satisfying a certain infsup condition. Examples are enriched simplicial finite elements and standard quadrilateral/hexahedral elements. The new approach overcomes the problem of an increasing discretization stencil and, thus, is simple to implement in existing computer codes. Numerical tests confirm the theoretical convergence results which are robust with respect to the user-chosen stabilization parameter.
\end{abstract}

\section{INTRODUCTION}

The standard Galerkin approach for solving the Stokes problem by finite element discretizations is well established [9, 17. If the finite element spaces approximating velocity and pressure satisfy an inf-sup condition, stability and convergence of the discretization can be proven. Applying this technique on adaptive meshes with hanging nodes, the additional constraints to guarantee the conformity of the velocity space reduce its dimension and it is not a priori clear whether the inf-sup condition remains valid for the considered pair of finite element spaces. Indeed, it is far from being trivial that the stability property proven on a family of regular meshes remains true when considering adaptive grids with hanging nodes [27.

An alternative approach for solving the Stokes problem consists of using equal order interpolations which are simple to implement since the same finite element space is used for approximating the pressure and the velocity components. However, we have to take into consideration that equal order interpolation does not satisfy the inf-sup stability condition and may produce oscillations in the pressure. Starting with [19, a methology has been developed to modify the discrete problem such that the correponding bilinear form is coercive on the product space. In this way stability is achieved for arbitrary pairs of finite elements including equal order interpolations on meshes with hanging nodes. Stability has been generated by adding weighted residuals of the strong form of the differential equation to the discrete problem. This pressure stabilized Petrov-Galerkin (PSPG) formulation has

Received by the editor April 17, 2007 and, in revised form, September 21, 2007.

2000 Mathematics Subject Classification. Primary 65N12; Secondary 65N30.

(C) 2008 American Mathematical Society Reverts to public domain 28 years from publication 
been successfully developed in different ways. Combined with the streamline upwind Petrov Galerkin (SUPG) stabilization, it has been used to treat two instability phenomena of the Navier-Stokes equations in a unique framework: the dominating advection for higher Reynolds numbers and the violation of the discrete inf-sup condition. The general idea behind this class of residual based stabilization technique is to enhance stability without upsetting consistency [14, 30. Moreover, it has been shown in 15, 18, 29, 30, that an additional least square term for the incompressibility condition, called grad-div stabilization, improves the robustness. Properties of the grad-div stabilization onto the algebraic solver are discussed in [26].

Another approach is the projection based stabilization technique. The stabilization by projecting the pressure gradient onto a continuous finite element space has been analyzed in [13. This method has been shown to be still consistent in the sense that a smooth exact solution satisfies the discrete problem. However, the method is quite expensive due to the nonlocal behavior of the projection. Alternatively, a two-level approach with a projection onto a discontinuous finite element space living on patches of elements has been analyzed in [4. This more local method is no longer consistent in a strong manner but the consistency error can be bounded by a rate which does not reduce the optimal order of convergence. The technique of local projection has been investigated to stabilize dominating advection for transport equations in 5 and extended to the Oseen equation in [7. A drawback of the two-level approach from the implementation point of view is the fact that the added stabilizing term produces a larger stencil which may not fit in the data structure of an available programming code. As pointed out in [23, the key point of stabilization by local projection is the existence of a special interpolant with additional orthogonality properties with respect to the projection space. This general view opens the possibility to construct stabilized methods for enriched equal order finite element spaces by local projection onto a discontinuous finite element space living on the same mesh. In particular, the added stabilizing term does not produce a larger stencil in contrast to the two-level approach.

From the implementational point of view, equal order interpolations allow us to work with only one finite element space, but additional stabilization terms have to be assembled. Note that the PSPG approach includes more stabilizing terms compared to the local projection method.

The main objective of this paper is to adapt this new approach to the Stokes problem, to give examples of solution and projection spaces allowing stability and optimal convergence, and to demonstrate the potential of the method by a number of selected numerical tests. The remaining part of the paper is organized as follows. In Section 2, a weak formulation of the Stokes problem and its standard Galerkin discretization is given. We formulate the local projection method for arbitrary equal order interpolations satisfying a certain compatibility condition with respect to the projection space. Section 3 is devoted to optimal a-priori estimates related to an asymptotically optimal choice of the stabilization parameter. In Section 4, we consider examples of enriched finite element spaces satisfying the compatibility condition. In particular, we show the equivalence of the projection based stabilization method of lowest order enriched finite elements on simplices with the PSPG/graddiv stabilization of the unenriched spaces. Finally, the properties of the method are illustrated by numerical tests in Section 5 . 
Notation. Throughout this paper, $C$ will denote a generic positive constant which is independent of the mesh. Subscripted constants such as $C_{1}$ are also independent of the mesh, but have a fixed value. We will write shortly $\alpha \sim \beta$, if there are positive constants $\underline{C}$ and $\bar{C}$ such that

$$
\underline{C} \beta \leq \alpha \leq \bar{C} \beta
$$

holds. The Stokes problem will be considered in the domain $\Omega \subset \mathbb{R}^{d}, d=2,3$, which is assumed to be a polygonal or polyhedral domain with boundary $\partial \Omega$. For a measurable subset $G$ of $\Omega$, the usual Sobolev spaces $W^{m, p}(G)$ with norm $\|\cdot\|_{m, p, G}$ and semi-norm $|\cdot|_{m, p, G}$ are used. In the case $p=2$, we have $H^{m}(G)=W^{m, 2}(G)$ and the index $p$ will be omitted. The $L^{2}$ inner product on $G$ is denoted by $(\cdot, \cdot)_{G}$. Note that the index $G$ will be omitted for $G=\Omega$. This notation of norms, semi-norms, and inner products is also used for the vector-valued and tensor-valued case.

\section{StOKES PROBlem AND ITS DisCRETIZATION}

2.1. Weak formulation. Let $\Omega \subset \mathbb{R}^{d}$ be a polygonal $(d=2)$ or polyhedral $(d=3)$ domain with Lipschitz continuous boundary $\Gamma=\partial \Omega$. We consider the Stokes problem

$$
\left.\begin{array}{rl}
-\triangle u+\nabla p=f & \text { in } \Omega, \\
\operatorname{div} u=0 & \text { in } \Omega, \\
u=0 & \text { on } \Gamma,
\end{array}\right\}
$$

where $f$ is a given body force, $u$ and $p$ denote the velocity and pressure field, respectively. Introducing the spaces $V:=\left(H_{0}^{1}(\Omega)\right)^{d}$ and $Q:=L_{0}^{2}(\Omega)$, a weak formulation of (2.1) reads:

Find $(u, p) \in V \times Q$ such that

$$
(\nabla u, \nabla v)-(p, \operatorname{div} v)+(q, \operatorname{div} u)=(f, v) \quad \forall(v, q) \in V \times Q .
$$

The Lax-Milgram theorem applied to the subspace of divergence-free functions and the inf-sup condition

$$
\inf _{q \in Q} \sup _{v \in V} \frac{(q, \operatorname{div} v)}{\|q\|_{0}|v|_{1}}>0
$$

guarantee that there is a unique solution of (2.2); see [17].

2.2. Discrete problem and stabilized formulation. We are given a family $\left\{\mathcal{T}_{h}\right\}$ of shape-regular decompositions of $\Omega$ into $d$-simplices, quadrilaterals, or hexahedra. The diameter of a cell $K$ is denoted by $h_{K}$. The mesh parameter $h$ describes the maximum diameter of the cells $K \in \mathcal{T}_{h}$.

Let $Y_{h}$ be a scalar finite element space of continuous, piecewise polynomials functions over $\mathcal{T}_{h}$. The spaces for approximating the velocity and the pressure are given by $V_{h}:=Y_{h}^{d} \cap V$ and $Q_{h}:=Y_{h} \cap Q$, respectively.

The standard Galerkin discretization reads:

Find $\left(u_{h}, p_{h}\right) \in V_{h} \times Q_{h}$ such that for all $\left(v_{h}, q_{h}\right) \in V_{h} \times Q_{h}$,

$$
\left(\nabla u_{h}, \nabla v_{h}\right)-\left(p_{h}, \operatorname{div} v_{h}\right)+\left(q_{h}, \operatorname{div} u_{h}\right)=\left(f, v_{h}\right) .
$$


It is known that, in general, equal order interpolations do not satisfy the discrete counterpart of (2.3), i.e., the Babuška-Brezzi condition [9]

$$
\exists \beta_{0}>0, \forall h: \quad \inf _{q_{h} \in Q_{h}} \sup _{v_{h} \in V_{h}} \frac{\left(q_{h}, \operatorname{div} v_{h}\right)}{\left\|q_{h}\right\|_{0}\left|v_{h}\right|_{1}} \geq \beta_{0}
$$

is violated. Therefore, we intend to add to (2.4) a stabilizing term based on local projection. To this end, we need further notation. Let $P_{s}(K), s=0,1, \ldots$, denote the set of all polynomials of degree less than or equal to $s$ and let $D_{h}(K)$ be a finite dimensional function space on the cell $K \in \mathcal{T}_{h}$ with $P_{s}(K) \subset D_{h}(K)$. We extend the definition by allowing $D_{h}(K)=\{0\}$ together with $P_{-1}(K)=D_{h}(K)$. We introduce the associated global space of discontinuous finite elements

$$
D_{h}:=\bigoplus_{K \in \mathcal{T}_{h}} D_{h}(K)
$$

and the local $L^{2}(K)$-projection $\pi_{K}: L^{2}(K) \rightarrow D_{h}(K)$ which generates the global projection $\pi_{h}: L^{2}(\Omega) \rightarrow D_{h}$ by

$$
\left.\left(\pi_{h} w\right)\right|_{K}:=\pi_{K}\left(\left.w\right|_{K}\right) \quad \forall K \in \mathcal{T}_{h}, \forall w \in L^{2}(\Omega) .
$$

The fluctuation operator $\kappa_{h}: L^{2}(\Omega) \rightarrow L^{2}(\Omega)$ is given by $\kappa_{h}:=i d-\pi_{h}$ where $i d: L^{2}(\Omega) \rightarrow L^{2}(\Omega)$ is the identity on $L^{2}(\Omega)$. We will have a closer look at the case $D_{h}(K)=\{0\}$. The inclusion $P_{s}(K) \subset D_{h}(K)$ is satisfied for $s=-1$. Moreover, since $\pi_{K} w=0$ for all $w \in L^{2}(K)$, the fluctuation operator $\kappa_{h}$ degenerates to the identity. For simplicity of notation, we will not distinguish between the scalar operators $i d, \pi_{K}, \pi_{h}, \kappa_{h}$ acting on scalar functions and their vector-valued versions. Thus, $\kappa_{h} \nabla p$ has to be understood as acting on each component of $\nabla p$ separately.

Now we are able to introduce the stabilizing term

$$
S_{h}\left(p_{h}, q_{h}\right):=\sum_{K \in \mathcal{T}_{h}} \alpha_{K}\left(\kappa_{h} \nabla p_{h}, \kappa_{h} \nabla q_{h}\right)_{K}
$$

where $\alpha_{K}$ are parameters to be chosen. Our stabilized discrete problem reads:

Find $\left(u_{h}, p_{h}\right) \in V_{h} \times Q_{h}$ such that for all $\left(v_{h}, q_{h}\right) \in V_{h} \times Q_{h}$,

$$
\left(\nabla u_{h}, \nabla v_{h}\right)-\left(p_{h}, \operatorname{div} v_{h}\right)+\left(q_{h}, \operatorname{div} u_{h}\right)+S_{h}\left(p_{h}, q_{h}\right)=\left(f, v_{h}\right) .
$$

In order to study the properties of (2.7) on the product space $V_{h} \times Q_{h}$, we introduce the bilinear form

$$
A_{h}((u, p) ;(v, q)):=(\nabla u, \nabla v)-(p, \operatorname{div} v)+(q, \operatorname{div} u)+S_{h}(p, q)
$$

and the mesh-dependent norm

$$
\|\mid(v, q)\| \|:=\left(|v|_{1}^{2}+\|q\|_{0}^{2}+\sum_{K \in \mathcal{T}_{h}} \alpha_{K}\left\|\kappa_{h} \nabla q\right\|_{0, K}^{2}\right)^{1 / 2} .
$$

\section{Stability And CONVERGEnCE}

We are interested in methods of a fixed order $r \in \mathbb{N}$. In order to guarantee stability and convergence of our method (2.7), we suppose that following assumptions are satisfied. 
Assumption A1. For the fluctuation operator $\kappa_{h}$, the approximation property

$$
\left\|\kappa_{h} w\right\|_{0, K} \leq C h_{K}^{\ell}|w|_{\ell, K} \quad \forall w \in H^{\ell}(K), \forall K \in \mathcal{T}_{h}, 0 \leq \ell \leq r-1
$$

holds.

Note that Assumption A1 is fulfilled in the case $P_{r-2}(K) \subset D_{h}(K)$ for any $r \in \mathbb{N}$. We have for $D_{h}(K)=\{0\}$ by definition $P_{-1}(K) \subset D_{h}(K)$ and

$$
\left\|\kappa_{h} w\right\|_{0, K}=\| \text { id } w\left\|_{0, K}=\right\| w \|_{0, K} \quad \forall w \in L^{2}(K), \forall K \in \mathcal{T}_{h} .
$$

In the case $\operatorname{dim}\left(D_{h}(K)\right) \geq 1$, Assumption A1 follows from the Bramble-Hilbert lemma.

Assumption A2. The finite element space $Y_{h}$ approximates $H^{1}(\Omega)$ of order $r$, i.e., there exists an interpolation operator $i_{h}: H^{1}(\Omega) \rightarrow Y_{h}$ such that $i_{h} v \in H_{0}^{1}(\Omega)$ for all $v \in H_{0}^{1}(\Omega)$ and the error estimate

$$
\left\|v-i_{h} v\right\|_{0, K}+h_{K}\left|v-i_{h} v\right|_{1, K} \leq C h_{K}^{\ell}\|v\|_{\ell, \omega(K)}
$$

holds for all $v \in H^{\ell}(\omega(K))$, for all $K \in \mathcal{T}_{h}$, and $1 \leq \ell \leq r+1$ where $\omega(K)$ denotes a certain local neighborhood of $K$ which appears in the definition of these interpolation operators for non-smooth functions; see [12, 28] for more details.

The existence theory of interpolation operators for non-smooth functions satisfying Assumption A2 is well established in the literature; see [1, 12, 28].

Assumption A3. There exists an interpolation operator $j_{h}: H^{1}(\Omega) \rightarrow Y_{h}$ with $j_{h} v \in H_{0}^{1}(\Omega)$ for all $v \in H_{0}^{1}(\Omega)$, the orthogonality property

$$
\left(w-j_{h} w, q_{h}\right)=0 \quad \forall q_{h} \in D_{h}, \forall w \in H^{1}(\Omega),
$$

and the approximation property

$$
\left\|w-j_{h} w\right\|_{0, K}+h\left|w-j_{h} w\right|_{1, K} \leq C h_{K}^{\ell}\|w\|_{\ell, \omega(K)},
$$

for all $w \in H^{\ell}(\omega(K)), 1 \leq \ell \leq r+1$, where $\omega(K)$ denotes again a certain local neighborhood of $K$.

Note that (3.1) holds for $D_{h}(K)=\{0\}$. For $\operatorname{dim} D_{h}(K) \geq 1$, the existence of an interpolation operator satisfying the additional orthogonality property (3.1) has been investigated in [23]. It turns out that the Assumption A2 together with the local inf-sup condition

$$
\exists \beta_{1}>0 \quad \forall h>0, \forall K \in \mathcal{T}_{h}: \quad \inf _{q_{h} \in D_{h}(K)} \sup _{v_{h} \in Y_{h}(K)} \frac{\left(v_{h}, q_{h}\right)_{K}}{\left\|v_{h}\right\|_{0, K}\left\|q_{h}\right\|_{0, K}} \geq \beta_{1}
$$

for the spaces $D_{h}(K)$ and $Y_{h}(K)$ are sufficient for A3; see [23, Theorem 2.2]. Here, $Y_{h}(K)$ is the restriction of functions from $Y_{h}$ with support in $\bar{K}$, i.e.,

$$
Y_{h}(K):=\left\{\left.w_{h}\right|_{K}: w_{h} \in Y_{h}, w_{h}=0 \text { on } \Omega \backslash K\right\} .
$$

The local inf-sup condition will be fulfilled if $Y_{h}(K)$ is rich enough compared to $D_{h}(K)$. Special pairs of spaces $Y_{h}(K)$ and $D_{h}(K)$ satisfying the local inf-sup condition will be studied in Section 4 .

Lemma 3.1. Let the Assumptions A1, A3, and $h_{K}^{2} / \alpha_{K} \leq C$ be fulfilled. Then, there exists a positive constant $\beta$ independent of $h$ such that

$$
\inf _{\left(v_{h}, q_{h}\right) \in V_{h} \times Q_{h}} \sup _{\left(w_{h}, r_{h}\right) \in V_{h} \times Q_{h}} \frac{A_{h}\left(\left(v_{h}, q_{h}\right) ;\left(w_{h}, r_{h}\right)\right)}{\left\|( v _ { h } , q _ { h } ) \left|\left\|||\left|\left(w_{h}, r_{h}\right) \|\right|\right.\right.\right.} \geq \beta
$$

holds. 
Proof. Let $\left(v_{h}, q_{h}\right)$ be an arbitrary element of $V_{h} \times Q_{h}$. We obtain

$$
A_{h}\left(\left(v_{h}, q_{h}\right) ;\left(v_{h}, q_{h}\right)\right)=\left|v_{h}\right|_{1}^{2}+\sum_{K \in \mathcal{T}_{h}} \alpha_{K}\left\|\kappa_{h} \nabla q_{h}\right\|_{0, K}^{2} .
$$

Furthermore, due to the continuous inf-sup condition (2.3), there exists for any $q_{h} \in Q_{h}$ an element $v_{q_{h}} \in V$ satisfying

$$
-\left(q_{h}, \operatorname{div} v_{q_{h}}\right)=\left\|q_{h}\right\|_{0}^{2}, \quad\left\|v_{q_{h}}\right\|_{1} \leq C\left\|q_{h}\right\|_{0} .
$$

Consequently, we have for the interpolant $j_{h} v_{q_{h}}$,

$$
\begin{array}{r}
A_{h}\left(\left(v_{h}, q_{h}\right) ;\left(j_{h} v_{q_{h}}, 0\right)\right)=-\left(q_{h}, \operatorname{div} j_{h} v_{q_{h}}\right)+\left(\nabla v_{h}, \nabla j_{h} v_{q_{h}}\right) \\
=\left\|q_{h}\right\|_{0}^{2}+\left(q_{h}, \operatorname{div}\left(v_{q_{h}}-j_{h} v_{q_{h}}\right)\right)+\left(\nabla v_{h}, \nabla j_{h} v_{q_{h}}\right) .
\end{array}
$$

Using integration by parts, the orthogonality property (3.1), and the assumption $h_{K}^{2} / \alpha_{K} \leq C$, we estimate the second term in (3.6) as follows:

$$
\begin{aligned}
\mid\left(q_{h}, \operatorname{div}\left(v_{q_{h}}\right.\right. & \left.\left.-j_{h} v_{q_{h}}\right)\right)|=|\left(\nabla q_{h}, v_{q_{h}}-j_{h} v_{q_{h}}\right)|=|\left(\kappa_{h} \nabla q_{h}, v_{q_{h}}-j_{h} v_{q_{h}}\right) \mid \\
& \leq C\left(\sum_{K \in \mathcal{T}_{h}} \alpha_{K}\left\|\kappa_{h} \nabla q_{h}\right\|_{0, K}^{2}\right)^{1 / 2}\left(\sum_{K \in \mathcal{T}_{h}} \frac{h_{K}^{2}}{\alpha_{K}}\left|v_{q_{h}}\right|_{1, K}^{2}\right)^{1 / 2} \\
& \leq C_{1}\left(\sum_{K \in \mathcal{T}_{h}} \alpha_{K}\left\|\kappa_{h} \nabla q_{h}\right\|_{0, K}^{2}\right)^{1 / 2}\left\|q_{h}\right\|_{0} \\
& \leq \frac{\left\|q_{h}\right\|_{0}^{2}}{4}+C_{1}^{2} \sum_{K \in \mathcal{T}_{h}} \alpha_{K}\left\|\kappa_{h} \nabla q_{h}\right\|_{0, K}^{2} .
\end{aligned}
$$

The last term in (3.6) is estimated by

$$
\left|\left(\nabla v_{h}, \nabla j_{h} v_{q_{h}}\right)\right| \leq\left|v_{h}\right|_{1}\left|j_{h} v_{q_{h}}\right|_{1} \leq C_{2}\left|v_{h}\right|_{1}\left\|q_{h}\right\|_{0} \leq \frac{\left\|q_{h}\right\|_{0}^{2}}{4}+C_{2}^{2}\left|v_{h}\right|_{1}^{2} .
$$

Summarizing the estimates of the individual terms, we end up with

$$
A_{h}\left(\left(v_{h}, q_{h}\right) ;\left(j_{h} v_{q_{h}}, 0\right)\right) \geq \frac{\left\|q_{h}\right\|_{0}^{2}}{2}-C_{3}\left(\left|v_{h}\right|_{1}^{2}+\sum_{K \in \mathcal{T}_{h}} \alpha_{K}\left\|\kappa_{h} \nabla q_{h}\right\|_{0, K}^{2}\right)
$$

where $C_{3}=\max \left(C_{1}^{2}, C_{2}^{2}\right)$. Multiplying this inequality by $2 /\left(1+2 C_{3}\right)$ and adding it to (3.5) we conclude that for any $\left(v_{h}, q_{h}\right) \in V_{h} \times Q_{h}$ there is $\left(w_{h}, r_{h}\right):=\left(v_{h}, q_{h}\right)+$ $2 /\left(1+2 C_{3}\right)\left(j_{h} v_{q_{h}}, 0\right)$ such that

$$
A_{h}\left(\left(v_{h}, q_{h}\right) ;\left(w_{h}, r_{h}\right)\right) \geq \frac{1}{1+2 C_{3}} \mid\left\|\left(v_{h}, q_{h}\right)\right\|^{2} .
$$

Furthermore, the $H^{1}$-stability of the interpolation $j_{h}$ and the definition of $v_{q_{h}}$ imply

$$
\begin{aligned}
\left\|\left|\left(w_{h}, r_{h}\right)\right|\right\| & \leq\left.\left\|\left|\left(v_{h}, q_{h}\right)\right|\right\|\left|+\frac{2}{1+2 C_{3}}\right| j_{h} v_{q_{h}}\right|_{1} \leq\left\|\left|\left(v_{h}, q_{h}\right)\right|\right\|+C_{4}\left\|q_{h}\right\|_{0} \\
& \leq\left(1+C_{4}\right)\left\|\mid\left(v_{h}, q_{h}\right)\right\| .
\end{aligned}
$$

Thus, the lemma holds with $\beta=\left(\left(1+2 C_{3}\right)\left(1+C_{4}\right)\right)^{-1}$.

Note, that Lemma 3.1 implies the unique solvability of the stabilized discrete problem.

Now we will consider the consistency error. 
Lemma 3.2. Assume that the fluctuation operator $\kappa_{h}$ satisfies Assumption A1. Let $(u, p) \in V \times\left(Q \cap H^{r}(\Omega)\right)$ be the solution of the Stokes problem (2.2) and $\left(u_{h}, p_{h}\right) \in V_{h} \times Q_{h}$ the solution of the stabilized problem (2.7). Then, the consistency error can be estimated by

$$
\left|A_{h}\left(\left(u-u_{h}, p-p_{h}\right) ;\left(v_{h}, q_{h}\right)\right)\right| \leq C\left(\sum_{K \in \mathcal{T}_{h}} \alpha_{K} h_{K}^{2 r-2}|p|_{r, K}^{2}\right)^{1 / 2}||\left|\left(v_{h}, q_{h}\right)\right|||
$$

for all $\left(v_{h}, q_{h}\right) \in V_{h} \times Q_{h}$.

Proof. Using (2.8), (2.2), and (2.7), we obtain

$$
A_{h}\left(\left(u-u_{h}, p-p_{h}\right) ;\left(v_{h}, q_{h}\right)\right)=S_{h}\left(p, q_{h}\right) .
$$

Using the approximation property of the fluctuation operator $\kappa_{h}$,

$$
\left\|\kappa_{h} \nabla p\right\|_{0, K} \leq C h_{K}^{r-1}|\nabla p|_{r-1, K} \leq C h_{K}^{r-1}|p|_{r, K}
$$

and Cauchy-Schwarz inequality, we end up with the statement of the lemma.

Theorem 3.3. Assume A1, A2, A3, and $\alpha_{K} \sim h_{K}^{2}$. Let the solution of (2.2) satisfy $(u, p) \in\left(V \cap H^{r+1}(\Omega)^{d}\right) \times\left(Q \cap H^{r}(\Omega)\right)$ and let $\left(u_{h}, p_{h}\right) \in V_{h} \times Q_{h}$ be the solution of (2.7). Then, there exists a positive constant $C$ independent of $h$ such that the error estimate

$$
\left\|\left(u-u_{h}, p-p_{h}\right) \mid\right\| \leq C h^{r}\left(\|u\|_{r+1}+\|p\|_{r}\right)
$$

holds true. Moreover, if the Stokes problem is $H^{2}(\Omega)^{d} \times H^{1}(\Omega)$-regular, there exists a positive constant $C$ independent of $h$ such that

$$
\left\|u-u_{h}\right\|_{0} \leq C h^{r+1}\left(\|u\|_{r+1}+\|p\|_{r}\right)
$$

holds.

Proof. Using Lemma 3.1, we can estimate

$$
\begin{aligned}
\left\|\left(j_{h} u-u_{h}, i_{h} p-p_{h}\right)\right\| \leq & \frac{1}{\beta} \sup _{\left(w_{h}, r_{h}\right) \in V_{h} \times Q_{h}} \frac{A_{h}\left(\left(j_{h} u-u_{h}, i_{h} p-p_{h}\right) ;\left(w_{h}, r_{h}\right)\right)}{\left\|\left|\left(w_{h}, r_{h}\right) \|\right|\right.} \\
\leq & \frac{1}{\beta} \sup _{\left(w_{h}, r_{h}\right) \in V_{h} \times Q_{h}} \frac{A_{h}\left(\left(u-u_{h}, p-p_{h}\right) ;\left(w_{h}, r_{h}\right)\right)}{\left\|\left|\left(w_{h}, r_{h}\right)\right|\right\|} \\
& +\frac{1}{\beta} \sup _{\left(w_{h}, r_{h}\right) \in V_{h} \times Q_{h}} \frac{A_{h}\left(\left(j_{h} u-u, i_{h} p-p\right) ;\left(w_{h}, r_{h}\right)\right)}{\left\|\left(w_{h}, r_{h}\right)\right\| \mid} .
\end{aligned}
$$

The consistency estimate of Lemma 3.2 and the choice of $\alpha_{K}$ yield

$$
\sup _{\left(w_{h}, r_{h}\right) \in V_{h} \times Q_{h}} \frac{A_{h}\left(\left(u-u_{h}, p-p_{h}\right) ;\left(w_{h}, r_{h}\right)\right)}{\left\|\left(w_{h}, r_{h}\right) \mid\right\|} \leq C h^{r}\|p\|_{r} .
$$

The terms in $A_{h}\left(\left(j_{h} u-u, i_{h} p-p\right) ;\left(w_{h}, r_{h}\right)\right)$ will be estimated individually. Employing the approximation properties of $j_{h}$ and $i_{h}$, we get

$$
\begin{aligned}
\left|\left(\nabla\left(j_{h} u-u\right), \nabla w_{h}\right)\right| & \leq\left|j_{h} u-u\right|_{1}\left|w_{h}\right|_{1} \leq C h^{r}\|u\|_{r+1}\left|\|\left(w_{h}, r_{h}\right)\right|||, \\
\left|\left(i_{h} p-p, \operatorname{div} w_{h}\right)\right| & \leq C\left\|i_{h} p-p\right\|_{0}\left|w_{h}\right|_{1} \leq C h^{r}\|p\|_{r}\left|\left\|\left(w_{h}, r_{h}\right) \mid\right\| .\right.
\end{aligned}
$$


For estimating the next term, we integrate by parts, apply the orthogonality property (3.1) of $j_{h}$, use $\alpha_{K} \sim h_{K}^{2}$, and obtain

$$
\begin{aligned}
& \left|\left(r_{h}, \operatorname{div}\left(j_{h} u-u\right)\right)\right|=\left|\left(\nabla r_{h}, j_{h} u-u\right)\right|=\left|\left(\kappa_{h} \nabla r_{h}, j_{h} u-u\right)\right| \\
& \quad \leq C\left(\sum_{K \in \mathcal{T}_{h}} \alpha_{K}\left\|\kappa_{h} \nabla r_{h}\right\|_{0, K}^{2}\right)^{1 / 2}\left(\sum_{K \in \mathcal{T}_{h}} \frac{h_{K}^{2}}{\alpha_{K}} h_{K}^{2 r}\|u\|_{r+1, \omega(K)}^{2}\right)^{1 / 2} \\
& \leq C h^{r}\|u\|_{r+1}\left\|\left|\left(w_{h}, r_{h}\right)\right|\right\| .
\end{aligned}
$$

The stabilizing term is estimated by taking into consideration Cauchy-Schwarz inequality, the $L^{2}$-stability of the fluctuation operator $\kappa_{h}$, the approximation properties of $i_{h}$, and $\alpha_{K} \sim h_{K}^{2}$ :

$$
\begin{aligned}
S_{h}\left(i_{h} p-p, r_{h}\right) & \leq\left(\sum_{K \in \mathcal{T}_{h}} \alpha_{K}\left\|\kappa_{h} \nabla\left(i_{h} p-p\right)\right\|_{0, K}^{2}\right)^{1 / 2}\left(\sum_{K \in \mathcal{T}_{h}} \alpha_{K}\left\|\kappa_{h} \nabla r_{h}\right\|_{0, K}^{2}\right)^{1 / 2} \\
& \leq C\left(\sum_{K \in \mathcal{T}_{h}} \alpha_{K} h_{K}^{2 r-2}\|p\|_{r, \omega(K)}^{2}\right)^{1 / 2}\|\|\left(w_{h}, r_{h}\right)\|\| \\
& \leq C h^{r}\|p\|_{r}\left\|\left(w_{h}, r_{h}\right)\right\| .
\end{aligned}
$$

Putting the above estimates together, we obtain

$$
\left\|\left(j_{h} u-u_{h}, i_{h} p-p_{h}\right) \mid\right\| \leq C h^{r}\left(\|u\|_{r+1}+\|p\|_{r}\right) .
$$

Now, the triangle inequality and the estimate of the interpolation error with respect to $\||\cdot|||$ prove the first error estimate.

Under the assumption that the Stokes problem is $H^{2}(\Omega)^{d} \times H^{1}(\Omega)$-regular, we know that for any $g \in L^{2}(\Omega)^{d}$ there is a unique solution $\left(u_{g}, p_{g}\right) \in\left(V \cap H^{2}(\Omega)^{d}\right) \times$ $\left(Q \cap H^{1}(\Omega)\right)$ of the problem.

Find $\left(u_{g}, p_{g}\right) \in V \times Q$ such that

$$
\left(\nabla v, \nabla u_{g}\right)-\left(p_{g}, \operatorname{div} v\right)+\left(q, \operatorname{div} u_{g}\right)=(g, v) \quad \forall(v, q) \in V \times Q,
$$

satisfying

$$
\left\|u_{g}\right\|_{2}+\left\|p_{g}\right\|_{1} \leq C\|g\|_{0} .
$$

Setting $v=u-u_{h}$ and $q=0$, we get the representation

$$
\left(g, u-u_{h}\right)=\left(\nabla\left(u-u_{h}\right), \nabla u_{g}\right)-\left(p_{g}, \operatorname{div}\left(u-u_{h}\right)\right) .
$$

It follows from (2.2) and (2.7) that

$$
\left(\nabla\left(u-u_{h}\right), \nabla w_{h}\right)=\left(p-p_{h}, \operatorname{div} w_{h}\right)=-\left(p-p_{h}, \operatorname{div}\left(u_{g}-w_{h}\right)\right) \quad \forall w_{h} \in V_{h}
$$

and

$$
\left(r_{h}, \operatorname{div}\left(u-u_{h}\right)\right)=S_{h}\left(p, r_{h}\right)-S_{h}\left(p-p_{h}, r_{h}\right) \quad \forall r_{h} \in Q_{h},
$$

thus, (3.7) can be written in the form

$$
\begin{aligned}
\left(g, u-u_{h}\right)= & \left(\nabla\left(u-u_{h}\right), \nabla\left(u_{g}-w_{h}\right)\right)-\left(p-p_{h}, \operatorname{div}\left(u_{g}-w_{h}\right)\right) \\
& -\left(p_{g}-r_{h}, \operatorname{div}\left(u-u_{h}\right)\right)+S_{h}\left(p-p_{h}, r_{h}\right)-S_{h}\left(p, r_{h}\right) .
\end{aligned}
$$


Choosing $\left(w_{h}, r_{h}\right) \in V_{h} \times Q_{h}$ as an interpolant of $\left(u_{g}, p_{g}\right)$, we obtain

$$
\begin{aligned}
\left|\left(\nabla\left(u-u_{h}\right), \nabla\left(u_{g}-w_{h}\right)\right)\right| & \leq C\left|u-u_{h}\right|_{1} h\left\|u_{g}\right\|_{2}, \\
\left|\left(p-p_{h}, \operatorname{div}\left(u_{g}-w_{h}\right)\right)\right| & \leq C\left\|p-p_{h}\right\|_{0} h\left\|u_{g}\right\|_{2}, \\
\mid\left(p_{g}-r_{h}, \operatorname{div}\left(u-u_{h}\right)\right) & \leq C\left|u-u_{h}\right|_{1} h\left\|p_{g}\right\|_{1}, \\
\left|S_{h}\left(p-p_{h}, r_{h}\right)\right| & \leq\left(S_{h}\left(p-p_{h}, p-p_{h}\right)\right)^{1 / 2}\left(S_{h}\left(r_{h}, r_{h}\right)\right)^{1 / 2} \\
& \leq C\left\|||\left(u-u_{h}, p-p_{h}\right) \mid\right\| h\left\|p_{g}\right\|_{1}, \\
\left|S_{h}\left(p, r_{h}\right)\right| & \leq\left(\sum_{K \in \mathcal{T}_{h}} \alpha_{K}\left\|\kappa_{h} \nabla p\right\|_{0, K}^{2}\right)^{1 / 2}\left(S_{h}\left(r_{h}, r_{h}\right)\right)^{1 / 2} \\
& \leq C h^{r}\|p\|_{r} h\left\|p_{g}\right\|_{1} .
\end{aligned}
$$

Here, we used $\alpha_{K} \sim h_{K}^{2}$, Assumption A1, and the $H^{1}$ stability of the interpolation $r_{h} \in Q_{h}$ of $p_{g} \in Q$ to estimate $S_{h}\left(r_{h}, r_{h}\right)$. Summarizing, we have

$$
\begin{aligned}
\left|\left(g, u-u_{h}\right)\right| & \leq C h^{r}\left(\|u\|_{r+1}+\|p\|_{r}\right) h\left(\left\|u_{g}\right\|_{2}+\left\|p_{g}\right\|_{1}\right) \\
& \leq C h^{r+1}\left[\left(\|u\|_{r+1}+\|p\|_{r}\right)\|g\|_{0},\right.
\end{aligned}
$$

and

$$
\left\|u-u_{h}\right\|_{0}=\sup _{g \in L^{2}(\Omega)} \frac{\left(g, u-u_{h}\right)}{\|g\|_{0}}
$$

gives the desired $L^{2}$ error estimate.

Remark 3.4. The classical PSPG-stabilization is based on adding a stabilizing term of the form

$$
\sum_{K \in \mathcal{T}_{h}} \tau_{K}(-\Delta u+\nabla p-f, \nabla q)_{K}
$$

For getting coercivity of the associated bilinear form, an upper bound $\tau_{K} \leq \tau_{0} h_{K}^{2}$ has assumed to be fulfilled where $\tau_{0}$ depends on the constant $\mu$ in the inverse inequality

$$
\left\|\Delta v_{h}\right\|_{0, K} \leq \mu h_{K}^{-1}\left|v_{h}\right|_{1, K} \quad \forall v_{h} \in V_{h} .
$$

This results in a conditionally stable method in the sense of [6] unless linear elements are used. The local projection schemes considered here are unconditionally stable in the sense that the corresponding bilinear form satisfies the inf-sup condition (3.4) for $\alpha_{K}=\alpha_{0} h_{K}^{2}$ with $0<\alpha_{0}<\infty$.

\section{ENRICHED EQUAL ORDER INTERPOLATION}

We consider in this section combinations of approximation and projection spaces satisfying the Assumptions A1-A3 leading to methods of order $r \geq 1$. In [4, 7, two-level approaches have been developed where the approximation space $Y_{h}$ consists of standard finite elements of order $r=1,2$ on triangular and quadrilateral meshes $\mathcal{T}_{h}$. A discontinuous finite element space of order $r-1=0,1$ on a coarser grid level $\mathcal{T}_{2 h}$ has been used as projection space $D_{h}$. Drawbacks of this approach are that its stabilizing term increases the size of the stencil and it requires data structures which might not be available in an existing computer code. The general framework developed in [23] opens the way to consider continuous approximation and discontinuous projection spaces which are defined on the same mesh. All examples given for the Oseen problem in [23, Section 4] could also be used for the 
Stokes problem. However, due to the missing advection term, the range of possible spaces $Y_{h}$ and $D_{h}$ is much larger, in particular, the difference of orders can be two (instead of one for the Oseen problem).

4.1. Simplicial elements. Let us briefly discuss first order methods. We start with continuous, piecewise linear finite elements $(r=1)$ for approximating velocity and pressure. Choosing $D_{h}=\{0\}$, the fluctuation operator $\kappa_{h}$ becomes the identity and we end up with the stabilized method.

Find $\left(u_{h}, p_{h}\right) \in V_{h} \times Q_{h}$ such that for all $\left(v_{h}, q_{h}\right) \in V_{h} \times Q_{h}$,

$$
\left(\nabla u_{h}, \nabla v_{h}\right)-\left(p_{h}, \operatorname{div} v_{h}\right)+\left(q_{h}, \operatorname{div} u_{h}\right)+\sum_{K \in \mathcal{T}_{h}} \alpha_{K}\left(\nabla p_{h}, \nabla q_{h}\right)_{K}=\left(f, v_{h}\right) .
$$

This nonconsistent method has already been studied in [10]. Let us enrich only the velocity space elementwise by the bubble functions

$$
b_{K}=(d+1)^{d+1} \prod_{i=1}^{d+1} \lambda_{i}
$$

where $\lambda_{i}, i=1, \ldots, d+1$, denote the barycentric coordinates associated with the cell $K$. Then, we get the Mini-element, shortly denoted by $P_{1}^{+} / P_{1}$, which satisfies the Babuška-Brezzi condition (2.5) and has not been stabilized [2]. However, enriching both the velocity and the pressure space, we obtain the equal order interpolation $P_{1}^{+} / P_{1}^{+}$which needs extra stabilization. We use a local projection onto the space of piecewise constant functions, i.e. $D_{h}=P_{0}$. These first order element pairs are illustrated in Figure 1:
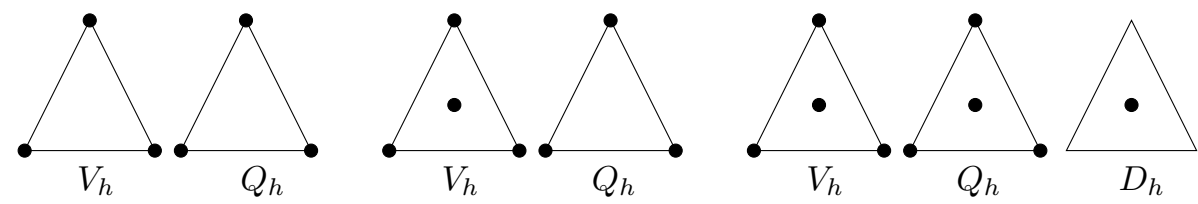

Figure 1. Examples of first order methods. Brezzi-Pitkäranta stabilization [10] $P_{1} / P_{1}$ (left), inf-sup stable Mini-element [2] $P_{1}^{+} / P_{1}$, local projection stabilization $P_{1}^{+} / P_{1}^{+} / P_{0}$ (right).

Lemma 4.1. Assume $Y_{h} / D_{h}=P_{1}^{+} / P_{0}$. Then, the assumptions $\mathrm{A} 1-\mathrm{A} 3$ are satisfied and the method (2.7) converges of first order for $\alpha_{K}=\alpha_{0} h_{K}^{2}, \alpha_{0}>0$.

Proof. A1 and A2 hold by construction. For showing A3, it suffices to prove the local inf-sup condition (3.3). Since $\operatorname{dim}\left(D_{h}(K)\right)=\operatorname{dim}\left(Y_{h}(K)\right)=1$, this is a simple task.

Now we will consider higher order methods on simplicial meshes, i.e., $r \geq 2$.

We enrich the space of polynomials of degree less than or equal to $r$ by bubble functions

$$
P_{r}^{b}(K):=P_{r}(K)+b_{K} \cdot P_{r-2}(K)
$$

and choose the approximation and projection spaces

$$
\begin{aligned}
Y_{h} & =\left\{v \in C(\bar{\Omega}):\left.v\right|_{K} \in P_{r}^{b}(K), \forall K \in \mathcal{T}_{h}\right\}, \\
D_{h} & =\left\{v \in L^{2}(\Omega):\left.v\right|_{K} \in P_{r-2}(K) \forall K \in \mathcal{T}_{h}\right\} .
\end{aligned}
$$


At first glance, the enriched space seems to be large, but a more careful look shows that

$$
P_{r}^{b}(K)=P_{r}(K) \oplus\left(b_{K} \cdot \sum_{i=2}^{d} \widetilde{P}_{r-i}(K)\right)
$$

where $\widetilde{P}_{m}(K)=\operatorname{span}\left(\left\{\prod_{i=1}^{d} x_{i}^{\alpha_{i}}, \sum_{i=1}^{d} \alpha_{i}=m\right\}\right)$.

Lemma 4.2. Assume $r \geq 2$ and $Y_{h} / D_{h}=P_{r}^{b} / P_{r-2}^{\text {disc }}$. Then, the Assumptions $\mathrm{A} 1-\mathrm{A} 3$ are satisfied and the method (2.7) converges of order $r$ for $\alpha_{K}=\alpha_{0} h_{K}^{2}$, $\alpha_{0}>0$.

Proof. A1 and A2 hold by construction. For showing A3, we prove the local inf-sup condition (3.3). Let $q_{h} \in D_{h}(K)=P_{r-2}(K)$ be arbitrary. We set $v_{h}:=b_{K} q_{h}$ and conclude $v_{h} \in P_{r}^{b}(K)$ where $v_{h}=0$ on $\partial K$. Thus, using the transformation onto a reference domain and the equivalence of norms on finite dimensional spaces, we have

$$
\left(q_{h}, v_{h}\right)_{K}=\left(b_{K} q_{h}, q_{h}\right)_{K} \geq C_{4}\left\|q_{h}\right\|_{0, K}^{2} .
$$

Moreover,

$$
\left\|v_{h}\right\|_{0, K}=\left\|b_{K} q_{h}\right\|_{0, K} \leq\left\|b_{K}\right\|_{\infty, K}\left\|q_{h}\right\|_{0, K}=\left\|q_{h}\right\|_{0, K},
$$

consequently, there exists for each $0 \neq q_{h} \in D_{h}(K)$ a $v_{h} \in Y_{h}(K)$ such that

$$
\frac{\left(q_{h}, v_{h}\right)_{K}}{\|q\|_{0, K}\left\|v_{h}\right\|_{0, K}} \geq C_{4}
$$

and the local inf-sup condition (3.3) is proven.

Remark 4.3. To fulfill the local inf-sup condition for the projection space $D_{h}=$ $P_{r-1}^{\text {disc }}$, we have to enrich the approximation space further:

$$
P_{r}^{b+}(K):=P_{r}^{b}(K) \oplus\left(b_{K} \cdot \widetilde{P}_{r-1}(K)\right) ;
$$

see 23. For $r=2$ and $d=2$, the two variants are indicated in Figure 2 using the notation $P_{2}^{b}$ and $P_{2}^{b+}$ for the enriched spaces with one and three additional bubbles, respectively.

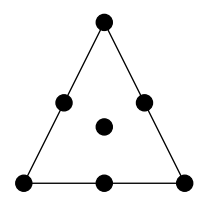

$V_{h}, Q_{h}$

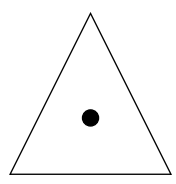

$D_{h}$

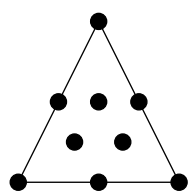

$V_{h}, Q_{h}$

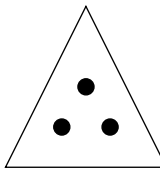

$D_{h}$

Figure 2. Examples of approximation and projection spaces of second order: $P_{2}^{b} / P_{0}$ (left) and $P_{2}^{b+} / P_{1}^{\text {disc }}$ (right). 
4.2. Quadrilateral and hexahedral elements. Now we consider the standard finite element spaces on regular families $\mathcal{T}_{h}$ of quadrilateral or hexahedral meshes [11. Let $\widehat{K}=(-1,+1)^{d}$ be the reference cell,

$$
Q_{r}(\widehat{K})=\left\{\hat{v}: \hat{v}(\hat{x})=\sum_{0 \leq \alpha_{i} \leq r} c_{\alpha_{1}, \ldots, \alpha_{d}} \hat{x}_{1}^{\alpha_{1}} \cdots \hat{x}_{d}^{\alpha_{d}},\right\},
$$

and $\hat{b}=\prod_{i=1}^{d}\left(1-\hat{x}_{i}^{2}\right) \in Q_{2}(\widehat{K})$ the bubble function on $\widehat{K}$. We denote by $F_{K}: \widehat{K} \rightarrow K$ the mapping from the reference cell onto the cell $K \in \mathcal{T}_{h}$ and set $v=\hat{v} \circ F_{K}^{-1}$. We introduce the finite element spaces

$$
\begin{aligned}
Q_{r} & :=\left\{v \in C(\bar{\Omega}):\left.v\right|_{K}=\hat{v} \circ F_{K}^{-1}, \hat{v} \in Q_{r}(\widehat{K}), \forall K \in \mathcal{T}_{h}\right\}, \quad r \geq 1, \\
Q_{r}^{\text {disc }} & :=\left\{v \in L^{2}(\Omega):\left.v\right|_{K}=\hat{v} \circ F_{K}^{-1}, \hat{v} \in Q_{r}(\widehat{K}), \forall K \in \mathcal{T}_{h}\right\}, \quad r \geq 0, \\
Q_{1}^{+} & :=\left\{v \in C(\bar{\Omega}):\left.v\right|_{K}=\hat{v} \circ F_{K}^{-1}, \hat{v} \in Q_{1}(\widehat{K})+\operatorname{span}(\hat{b}), \forall K \in \mathcal{T}_{h}\right\} .
\end{aligned}
$$

A first order method can be derived by setting $Y_{h}=Q_{1}$ and $D_{h}=\{0\}$, this results in the stabilized method (4.1). Note that in this case no projection is used.

Examples of enrichments of the velocity space such that the enriched velocity space together with the $Q_{1}$ pressure space satisfies the Babuška-Brezzi condition has been given in [3, 25, 16]. See 21] for a more general investigation. It should be mentioned that the local bubble space for enriching the $Q_{1}$ quadrilateral space has at least dimension 3 , more precisely, one additional degree of freedom for each component of the velocity and one degree of freedom shared by both components. This is true in the conforming [3] as well as in the nonconforming [16] case.

We turn back to equal order interpolations stabilized by local projection. For a local projection onto $D_{h}=Q_{0}=Q_{0}^{\text {disc }}$, we enrich the approximation space and set $Y_{h}=Q_{1}^{+}$.

Lemma 4.4. Assume $Y_{h} / D_{h}=Q_{1}^{+} / Q_{0}$. Then, the Assumptions $\mathrm{A} 1-\mathrm{A} 3$ are satisfied and method (2.7) converges of first order for $\alpha_{K}=\alpha_{0} h_{K}^{2}, \alpha_{0}>0$.

Proof. A1 and A2 hold by construction. For showing A3, it is enough to prove the local inf-sup condition (3.3). Since $\operatorname{dim}\left(D_{h}(K)\right)=\operatorname{dim}\left(Y_{h}(K)\right)=1$, this is a simple task.

We now turn to higher order methods.

Lemma 4.5. Assume $r \geq 2$ and $Y_{h} / D_{h}=Q_{r} / Q_{r-2}^{\text {disc }}$. Then, the Assumptions A1-A3 are satisfied and method (2.7) converges of order $r$ for $\alpha_{K}=\alpha_{0} h_{K}^{2}, \alpha_{0}>0$.

Proof. A1 and A2 hold by construction. For showing A3, we prove the local inf-sup condition (3.3). Let $q_{h} \in D_{h}(K)$ be arbitrary. We set $v_{h}:=\left(\hat{b} \circ F_{K}^{-1}\right) \cdot q_{h}$. Since $v_{h} \circ F_{K}=\hat{b} \cdot\left(q_{h} \circ F_{K}\right)$ and $q_{h} \circ F_{K} \in Q_{r-2}(\widehat{K})$, we conclude $v_{h} \in Y_{h}(K)$. Moreover, it follows from

$$
\left\|v_{h}\right\|_{0, K} \leq\left\|q_{h}\right\|_{0, K}
$$

that

$$
\frac{\left(q_{h}, v_{h}\right)_{K}}{\left\|q_{h}\right\|_{0, K}\left\|v_{h}\right\|_{0, K}} \geq \frac{\int_{\widehat{K}} \hat{b} \hat{q}_{h}^{2}\left|\operatorname{det} D F_{K}\right| d \hat{x}}{\int_{\widehat{K}} \hat{q}_{h}^{2}\left|\operatorname{det} D F_{K}\right| d \hat{x}} .
$$


Now, using the upper and lower bound

$$
C_{5} h_{K}^{d} \leq\left|\operatorname{det} D F_{K}\right| \leq C_{6} h_{K}^{d}
$$

shown in 24] and the equivalence of the norms

$$
\hat{q} \mapsto\left(\int_{\widehat{K}} \hat{q}^{2} d \hat{x}\right)^{1 / 2}, \quad \hat{q} \mapsto\left(\int_{\widehat{K}} \hat{b} \cdot \hat{q}^{2} d \hat{x}\right)^{1 / 2}
$$

on the finite dimensional space $Q_{r-2}(\widehat{K})$, we get the assertion of the lemma.

Remark 4.6. Note that for $r \geq 2$ and quadrilateral or hexahedral elements we do not need any enrichments. Thus, the standard finite element spaces can be used.

Remark 4.7. The smallest projections space which guarantees Assumption A1 is the space $P_{r-2}^{\text {disc }}$. Note that there are two versions of this space. The unmapped version is defined on the original cell $K \in \mathcal{T}_{h}$ by

$$
P_{r-2}^{\text {disc, } u}:=\left\{v:\left.v\right|_{K} \in P_{r-2}(K)\right\}
$$

and satisfies the usual approximation properties on shape regular meshes. Using the mapping $F_{K}: \widehat{K} \rightarrow K$ from the reference cell $(-1,+1)^{d}$ onto $K \in \mathcal{T}_{h}$, the mapped version is defined by

$$
P_{r-2}^{\text {disc, } m}:=\left\{v:\left.v\right|_{K} \circ F_{K} \in P_{r-2}(\widehat{K})\right\} .
$$

It satisfies the usual approximation properties only on families of successively refined quadrilateral and hexahedral meshes; see 22 for more details. Since both spaces are subspaces of $Q_{r-2}^{\text {disc }}$, the local inf-sup condition (3.3) is satisfied and Assumption A3 follows.

Remark 4.8. The local projection method with the larger projection space $D_{h}=$ $P_{r-1}^{\text {disc }}$ was analyzed in [23. In order to fulfill the local inf-sup condition, the approximation space $Y_{h}$ has to be enriched to

$$
Q_{r}^{b}:=\left\{v \in C(\bar{\Omega}):\left.v\right|_{K}=\hat{v} \circ F_{K}^{-1}, \hat{v} \in Q_{r}^{b}(\widehat{K}), \forall K \in \mathcal{T}_{h}\right\}
$$

where

$$
Q_{r}^{b}(\widehat{K}):=Q_{r}(\widehat{K}) \oplus \operatorname{span}\left(\hat{b} \hat{x}_{i}^{r-1}: i=1, \ldots, d\right) .
$$

Note that $Q_{r}^{b}(\widehat{K})$ contains for any $r \geq 2$ exactly $d$ functions more than $Q_{r}(\widehat{K})$ where $d$ is the space dimension.

4.3. Elimination of enrichments. In the examples above, standard finite element spaces have been enriched with bubble functions. These additional degrees of freedom can be eliminated locally by static condensation. We concentrate in this section on the special case $V_{h} / Q_{h} / D_{h}=P_{1}^{+} / P_{1}^{+} / P_{0}$ on simplices, i.e., our approximation space consists of continuous, piecewise linear functions enriched by one bubble function $b_{K}$ per cell:

$$
Y_{h}=\left\{v \in C(\bar{\Omega}):\left.v\right|_{K} \in P_{1}(K) \oplus \operatorname{span} b_{K}, \forall K \in \mathcal{T}_{h}\right\} .
$$

We set $V_{h}:=\left(Y_{h} \cap H_{0}^{1}(\Omega)\right)^{d}$ and $Q_{h}:=Y_{h} \cap L_{0}^{2}(\Omega)$. The stabilized discrete problem (2.7) can be written in the form:

Find $\left(u_{h}, p_{h}\right) \in V_{h} \times Q_{h}$ such that

$$
\begin{aligned}
\left(\nabla u_{h}, \nabla v_{h}\right)-\left(p_{h}, \operatorname{div} v_{h}\right) & =\left(f, v_{h}\right) & & \forall v_{h} \in V_{h}, \\
\left(q_{h}, \operatorname{div} u_{h}\right)+S_{h}\left(p_{h}, q_{h}\right) & =0 & & \forall q_{h} \in Q_{h} .
\end{aligned}
$$


Let us denote by $\langle\cdot, \cdot\rangle_{\partial G}$ the inner product in $L^{2}(\partial G)$ and by $n$ the outer unit normal with respect to $\Omega$. Since

$$
\left(1, \operatorname{div} u_{h}\right)+S_{h}\left(p_{h}, 1\right)=\left\langle 1, u_{h} \cdot n\right\rangle_{\partial \Omega}+\sum_{K \in \mathcal{T}_{h}} \alpha_{K}\left(\kappa_{h} \nabla p_{h}, \kappa_{h} \nabla 1\right)_{K}=0,
$$

the equation (4.3) is true for all $q_{h} \in Y_{h}$.

According to the splitting of the spaces $V_{h}$ and $Y_{h}$ into a linear and a bubble part

$$
V_{h}=V_{L} \oplus V_{B}, \quad Y_{h}=Y_{L} \oplus Y_{B},
$$

we split the functions $v_{h} \in V_{h}$ and $q_{h} \in Y_{h}$ into

$$
v_{h}=v_{L}+v_{B}, \quad q_{h}=q_{L}+q_{B} .
$$

Next, we decompose the stabilized discrete problem by using test functions from the linear and bubble part, respectively. This results in:

Find $\left(u_{L}, p_{L}\right) \in V_{L} \times Y_{L}$ and $\left(u_{B}, p_{B}\right) \in V_{B} \times Y_{B}$ such that $p_{L}+p_{B} \in Q_{h}$ and

$$
\left(\nabla u_{L}, \nabla v_{L}\right)+\left(\nabla u_{B}, \nabla v_{L}\right)-\left(p_{L}, \operatorname{div} v_{L}\right)-\left(p_{B}, \operatorname{div} v_{L}\right)=\left(f, v_{L}\right) \quad \forall v_{L} \in V_{L},
$$

$$
S_{h}\left(p_{L}+p_{B}, q_{L}\right)+\left(q_{L}, \operatorname{div} u_{L}\right)+\left(q_{L}, \operatorname{div} u_{B}\right)=0 \quad \forall q_{L} \in Y_{L},
$$

$\left(\nabla u_{L}, \nabla v_{B}\right)+\left(\nabla u_{B}, \nabla v_{B}\right)-\left(p_{L}, \operatorname{div} v_{B}\right)-\left(p_{B}, \operatorname{div} v_{B}\right)=\left(f, v_{B}\right) \quad \forall v_{B} \in V_{B}$,

$$
S_{h}\left(p_{L}+p_{B}, q_{B}\right)+\left(q_{B}, \operatorname{div} u_{L}\right)+\left(q_{B}, \operatorname{div} u_{B}\right)=0 \quad \forall q_{B} \in Y_{B} .
$$

A careful look shows that several terms in the discrete formulation vanish,

$$
\left(\nabla u_{B}, \nabla v_{L}\right)=\sum_{K \in \mathcal{T}_{h}}\left(\nabla u_{B}, \nabla v_{L}\right)_{K}=\sum_{K \in \mathcal{T}_{h}}\left[\left\langle\frac{\partial v_{L}}{\partial n_{K}}, u_{B}\right\rangle_{\partial K}-\left(\triangle v_{L}, u_{B}\right)_{K}\right]=0
$$

due to $\left.\Delta v_{L}\right|_{K}=0$ and $\left.u_{B}\right|_{\partial K}=0$. Analogously, $\left(\nabla u_{L}, \nabla v_{B}\right)=0$. It follows from

$$
\left(b_{K}, \frac{\partial b_{K}}{\partial x_{i}}\right)_{K}=\frac{1}{2} \int_{K} \frac{\partial b_{K}^{2}}{\partial x_{i}} d x=\frac{1}{2} \int_{\partial K} b_{K}^{2} n_{i} d \gamma=0, \quad i=1, \ldots, d,
$$

that

$$
\left(p_{B}, \operatorname{div} v_{B}\right)=\left(q_{B}, \operatorname{div} u_{B}\right)=0 .
$$

Furthermore, since $\nabla\left(\left.v_{L}\right|_{K}\right) \in P_{0}(K)$, we have $\pi_{h} \nabla v_{L}=\nabla v_{L}$. Consequently, $\kappa_{h} \nabla v_{L}=0$ such that $S_{h}\left(p_{L}+p_{B}, q_{L}\right)=0$ and $S_{h}\left(p_{L}+p_{B}, q_{B}\right)=S_{h}\left(p_{B}, p_{B}\right)$. Now, the bubble part of our discrete system reduces to:

Find $\left(u_{B}, p_{B}\right) \in V_{B} \times Y_{B}$ such that

$$
\begin{aligned}
\left(\nabla u_{B}, \nabla v_{B}\right)-\left(p_{L}, \operatorname{div} v_{B}\right) & =\left(f, v_{B}\right) & & \forall v_{B} \in V_{B}, \\
S_{h}\left(p_{B}, q_{B}\right)+\left(q_{B}, \operatorname{div} u_{L}\right) & =0 . & & \forall q_{B} \in Y_{B} .
\end{aligned}
$$

We extend the bubble function $b_{K}: K \rightarrow \mathbb{R}$ onto $\bar{\Omega}$ by setting $b_{K}=0$ outside of $\bar{K}$. Then, using the basis representations

$$
u_{B}=\sum_{K \in \mathcal{T}_{h}} u_{K} b_{K}, \quad p_{B}=\sum_{K \in \mathcal{T}_{h}} p_{K} b_{K},
$$

we can locally solve the bubble part of the algebraic system and get

$$
p_{K}=-\frac{\left(b_{K}, \operatorname{div} u_{L}\right)}{S_{h}\left(b_{K}, b_{K}\right)}, \quad\left(u_{K}\right)_{i}=\frac{1}{\left|b_{K}\right|_{1, K}^{2}}\left(f_{i}-\frac{\partial p_{L}}{\partial x_{i}}, b_{K}\right), \quad i=1, \ldots, d .
$$

Elimination of $u_{B}$ and $p_{B}$ in the linear part of the algebraic system yields 
Find $\left(u_{L}, p_{L}\right) \in V_{L} \times Y_{L}$ such that for all $\left(v_{L}, q_{L}\right) \in V_{L} \times Y_{L}$,

$$
\begin{aligned}
\left(\nabla u_{L}, \nabla v_{L}\right)-\left(p_{L}, \operatorname{div} v_{L}\right)+\sum_{K \in \mathcal{T}_{h}} \gamma_{K}\left(\operatorname{div} u_{L}, \operatorname{div} v_{L}\right) & =\left(f, v_{L}\right) \\
\left(q_{L}, \operatorname{div} u_{L}\right)+\sum_{K \in \mathcal{T}_{h}}\left(-\Delta u_{L}+\nabla p_{L}, \tau_{K} \nabla q_{L}\right)_{K} & =\sum_{K \in \mathcal{T}_{h}}\left(f, \tau_{K} \nabla q_{L}\right)_{K}
\end{aligned}
$$

where

$$
\gamma_{K}=\frac{1}{|K|} \frac{\left\|b_{K}\right\|_{0,1, K}^{2}}{S_{h}\left(b_{K}, b_{K}\right)}=\frac{\left\|b_{K}\right\|_{0,1, K}^{2}}{\alpha_{K}|K|\left\|\kappa_{h} \nabla b_{K}\right\|_{0, K}^{2}}, \quad \alpha_{K}(x)=\frac{\left\|b_{K}\right\|_{0,1, K}}{\left|b_{K}\right|_{1, K}^{2}} b_{K}(x) .
$$

Of course the pressure $p_{L} \in Y_{L}$ is only determined up to an additive constant. We make the pressure unique by replacing $Y_{L}$ by $Q_{L}=Y_{L} \cap L_{0}^{2}(\Omega)$. It is remarkable that the reduced problem corresponds to the PSPG method [19] in combination with the grad-div stabilization [15, 18, 29, 30].

Let us study the dependency of the parameter $\gamma_{K}$ and $\tau_{K}$ on $h_{K}$. We have

$$
\left\|b_{K}\right\|_{0,1, K}=\frac{(d+1)^{d+1} d !}{(2 d+1) !}|K|, \quad\left|b_{K}\right|_{1, K}^{2} \sim h_{K}^{-2}|K|, \quad\left\|\kappa_{h} \nabla b_{K}\right\|_{0, K}^{2} \sim h_{K}^{-2}|K|
$$

from which with the standard choice $\alpha_{K}=\alpha_{0} h_{K}^{2}$ follows:

$$
\gamma_{K} \sim \alpha_{K}^{-1} h_{K}^{2} \sim 1, \quad \tau_{K}(x) \sim h_{K}^{2} b_{K}(x) .
$$

Starting with the solution $\left(u_{L}, p_{L}\right) \in V_{L} \times Q_{L}$, we can reconstruct a velocity field

$$
u_{h}=u_{L}+u_{B}=u_{L}+\sum_{K \in \mathcal{T}_{h}} \frac{1}{\left|b_{K}\right|_{1, K}^{2}}\left(f-\nabla p_{L}, b_{K}\right) b_{K}
$$

satisfying the incompressibility constraint in the sense that

$$
\left(q_{L}, \operatorname{div} u_{h}\right)=0 \quad \forall q_{L} \in Q_{L} .
$$

\section{Numerical tests}

This section presents numerical results for solving the Stokes problem with equal order spaces where the discretization is stabilized by the local projection method. All calculations were performed with the code MooNMD [20] on a Linux PC (Pentium IV, $2.8 \mathrm{GHz})$.

Let $\Omega=(0,1)^{2}$. We consider the Stokes problem

$$
-\Delta u+\nabla p=f, \quad \operatorname{div} u=0 \quad \text { in } \Omega, \quad u=g \quad \text { on } \Gamma,
$$

where the right hand side $f$ and the inhomogeneous Dirichlet boundary condition $g$ have been chosen such that

$$
u=(\sin (x) \sin (y), \cos (x) \cos (y)), \quad p=2 \cos (x) \sin (y)-2 \sin (1)(1-\cos (1))
$$

is the solution. This example was taken from [8].

We have carried out calculations on triangular and quadrilateral meshes which were obtained by successive regular refinement of the initial coarse meshes. The coarsest mesh (level 0) consists of either two triangles or a single quadrilateral. The meshes on level 1 are shown in Figure 3 .

In the following, we evaluate the results of our calculations by considering the $L^{2}$ norm and the $H^{1}$ semi-norm of the velocity error, the $L^{2}$ norm of the pressure error, and the error in the local projection norm $\||\cdot|\|$. 

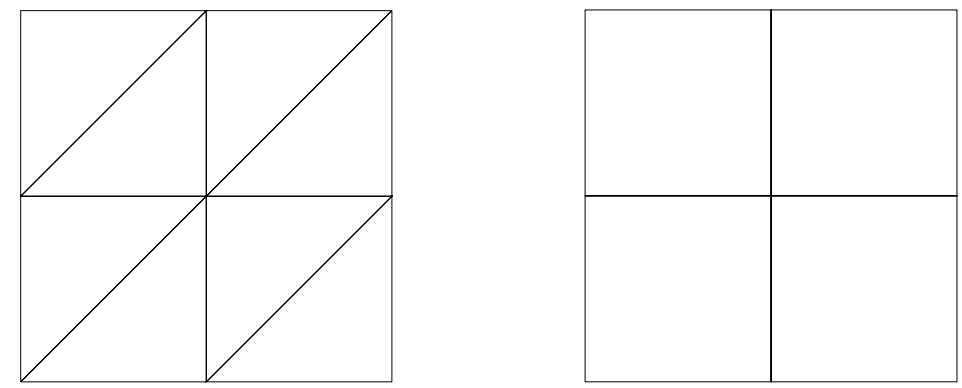

Figure 3. Meshes on level 1 consisting of triangles (left) and quadrilaterals (right).

The first group of calculations confirms predicted convergence rates. Furthermore, the results on triangular and quadrilateral meshes are compared. The parameter $\alpha_{0}$ is kept fixed for these calculations.

We start with first order elements on triangles and quadrilaterals. We consider the element $P_{1}^{+}$with projection onto $P_{0}$ on triangles, the Mini-element on triangles, and the element $Q_{1}^{+}$with projection onto $P_{0}$. The results of the Mini-element and the element $P_{1}^{+}$are almost the same although the Mini-element uses a smaller pressure space. Hence, the behavior of the error with respect to different norms is shown in Figure 4 only for the element $P_{1}^{+}$and the element $Q_{1}^{+}$. We have chosen for these calculations the parameter $\alpha_{0}=1$. The errors for the quadrilateral element are always smaller than the corresponding errors for the triangular elements. The largest difference appears for the errors in the local projection norm and for the $L^{2}$ norm of the pressure. The results differ by a factor of roughly 10. Furthermore, the $H^{1}$ semi-norm of the velocity error and the error in the local projection norm indicate, as predicted, first order convergence on both triangles and quadrilaterals. Moreover, the $L^{2}$ norm of the velocity error converges with second order on both types of meshes. The numerical results show that the convergence order for the $L^{2}$ norm of the pressure error is on both triangular and quadrilateral meshes approximately $3 / 2$ while our theory proves just first order convergence. A reason for this behavior might be some superconvergence phenomena for the first order elements.

Higher order elements are investigated next. Figure 5 presents the results for the third order elements $P_{3}^{b+}$ with projection onto $P_{2}^{\text {disc }}$ on triangles and $Q_{3}^{b}$ with projection onto $P_{2}^{\text {disc }}$ on quadrilaterals. Furthermore, the predicted asymptotic behavior is shown in the graphs of Figure 5. For these calculations, we have again chosen $\alpha_{K}=h_{K}^{2}$, i.e., $\alpha_{0}=1$. The $L^{2}$ norm of the pressure error, the $H^{1}$ semi-norm of the velocity error, and the error in the local projection norm converge with third order which confirms our theory. For these third order elements, there is no longer a better convergence of the $L^{2}$ norm of the pressure error as observed for the first order elements. The results for the $L^{2}$ norm of the velocity error indicate fourth order accuracy, in agreement with Theorem 3.3. The calculations on quadrilateral meshes result in a smaller error than the calculations on triangular meshes. For the velocity error, this effect is more pronounced for higher order elements.

We will study the behavior of different third order elements on quadrilaterals. We consider the following four elements: $Q_{3}^{b}$ with projection onto $P_{2}^{\text {disc }}, Q_{3}^{b}$ with 

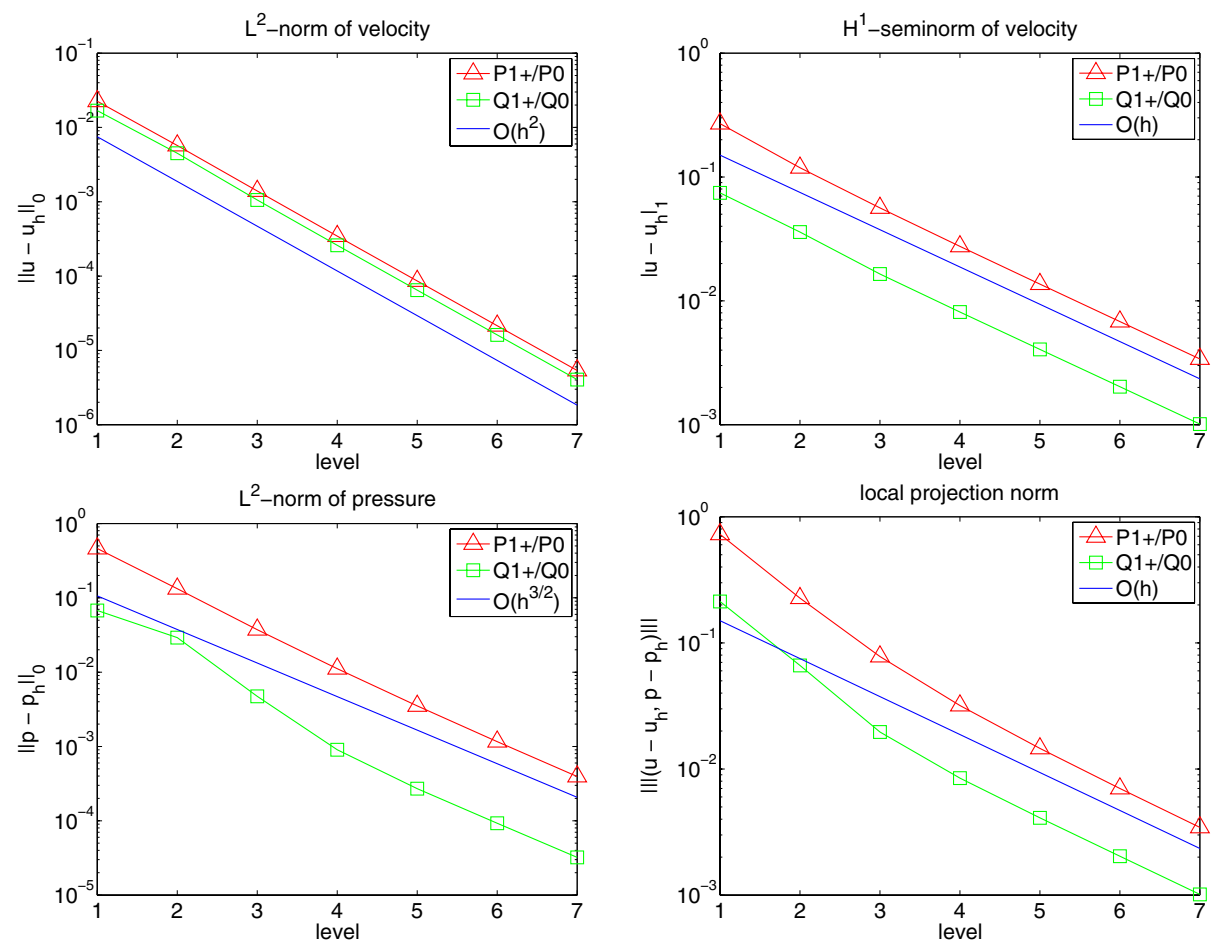

FiguRE 4. First order elements on triangles and quadrilaterals.

projection onto $P_{1}^{\text {disc }}, Q_{3}$ with projection onto $P_{1}^{\text {disc }}$, and $Q_{3}$ with projection onto $Q_{1}^{\text {disc }}$. The presented theory predicts for all four elements the same asymptotic convergence behavior. The parameter $\alpha_{0}$ was again chosen to be 1 .

The graphs in Figure 6 show that the element $Q_{3}^{b}$ with projection onto $P_{2}^{\text {disc }}$ gives the best results which are 1-2 order of magnitude better than the results of the other three elements. The results for the two elements which are projected onto $P_{1}^{\text {disc }}$ differ so slightly that the difference cannot be seen in the graphs. Looking at these results, we find that a larger projection space produces smaller errors. This is also indicated by the fact that the errors for the element which is projected onto $Q_{1}^{\text {disc }}$ are between the errors for the elements with projection space $P_{1}^{\text {disc }}$ and the element with projection space $P_{2}^{\text {disc }}$.

Next we consider how the results depend on the parameter $\alpha_{0}$ in the stabilization term. To this end, we have chosen three different cases: the first order element $P_{1}^{+}$ with projection onto $P_{0}$ on triangles, the third order element $Q_{3}^{b}$ with projection onto $P_{2}^{\text {disc }}$ on quadrilaterals, and the third order element $Q_{3}$ with projection onto $P_{1}^{\text {disc }}$.

Let us start with the first order element $P_{1}^{+}$with projection onto $P_{0}$ on triangles. The graphs in Figure 7 show the results on level 5 (2,048 triangles, 9,411 degrees of freedom for pressure and both velocity components), level 6 (8,192 triangles, 37,251 degrees of freedom), and level 7 (32,768 triangles, 148,227 degrees 

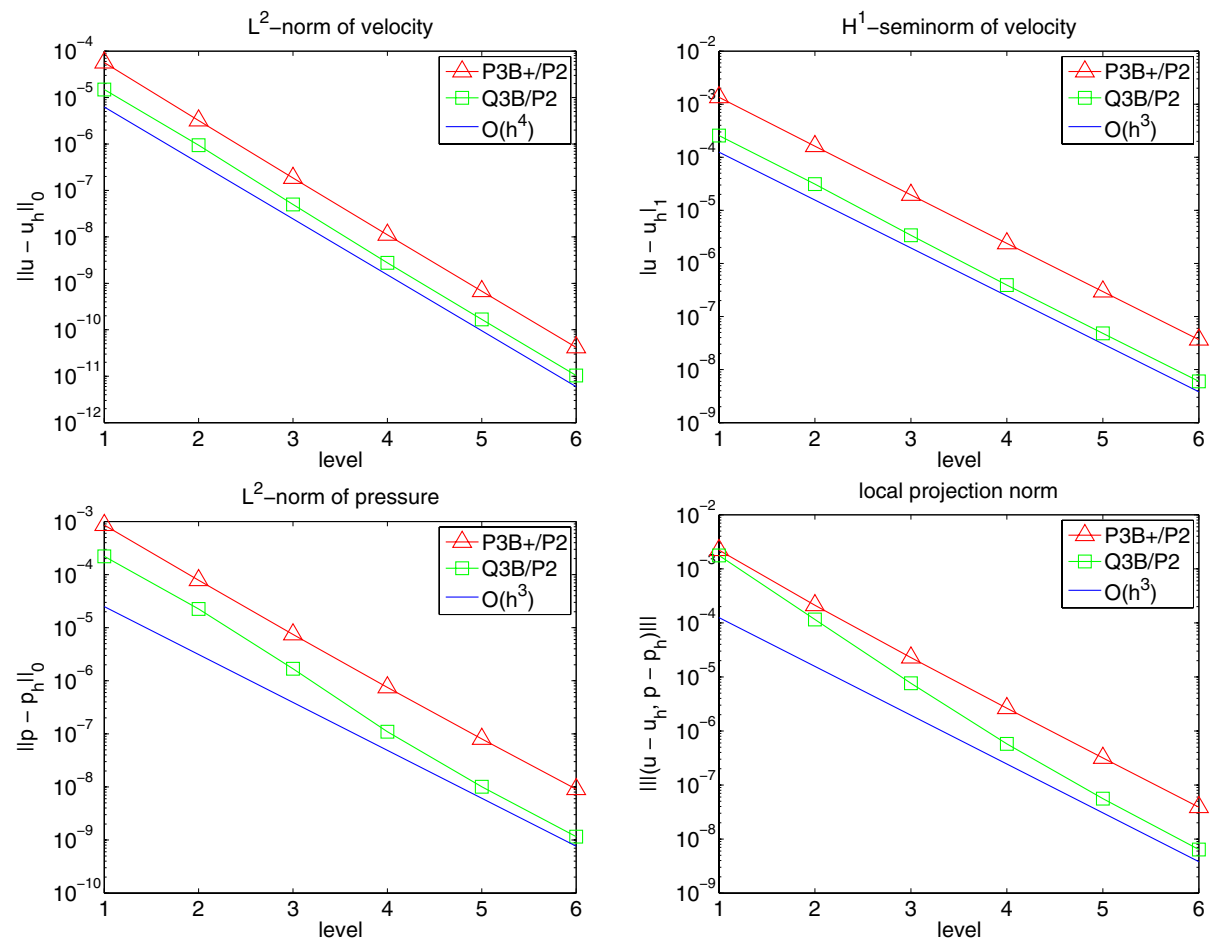

FiguRE 5. Third order elements on triangles and quadrilaterals.

of freedom). These results indicate that the velocity error is not very sensitive with respect to the parameter $\alpha_{0}$; see the top row of graphs in Figure 7 and note the scaling of the axes. The behavior of the pressure error and the error in the local projection norm is different. If the parameter $\alpha_{0}$ tends to zero then the error increases. This shows the instability of the equal order interpolation for the Stokes problem without stabilization. For each norm, the dependence of the error on the parameter $\alpha_{0}$ is very similar on the different refinement levels.

We continue by considering the third order element $Q_{3}^{b}$ with projection onto $P_{2}^{\text {disc }}$ on quadrilaterals. While varying the coefficient $\alpha_{0}$ in $\alpha_{K}=\alpha_{0} h_{K}^{2}$ over the wide range from $10^{-4}$ up to $10^{4}$, the obtained results changed only slightly. Furthermore, the behavior is similar on different refinement levels. If the parameter $\alpha_{0}$ tends towards zero in these calculations, then the error in the $L^{2}$ norm of the pressure and the error in the local projection norm increase while the velocity error remains bounded. This shows again the instability of equal order interpolation pairs for the Stokes problem.

Finally, we look at the element $Q_{3}$ with projection onto $P_{1}^{\text {disc }}$, i.e., the order difference between approximation space and projection space is 2 . The graphs of Figure 8 present the results on level 3 (64 quadrilaterals, 1,875 degrees of freedom for velocity and pressure), level 4 (256 quadrilaterals, 8,739 degrees of freedom), and level 5 (1,024 quadrilaterals, 28,227 degrees of freedom). These results show that this element is on all refinement levels much more sensitive with respect to the parameter $\alpha_{0}$ as the previously considered element $Q_{3}^{b}$ with projection onto $P_{2}^{\text {disc }}$. The error changes several orders of magnitude if $\alpha_{0}$ is varying. 

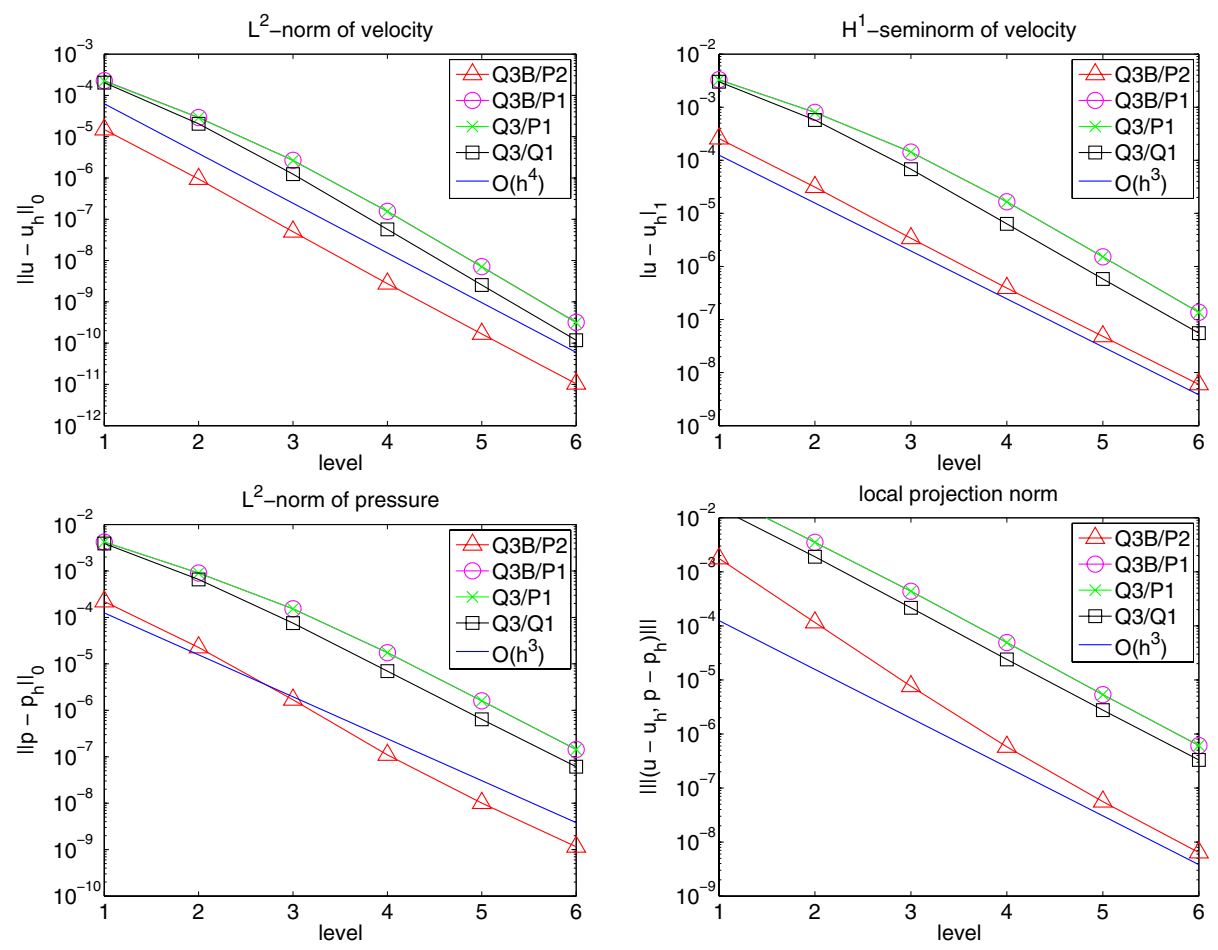

FiguRE 6. Comparison of third order elements on quadrilaterals.
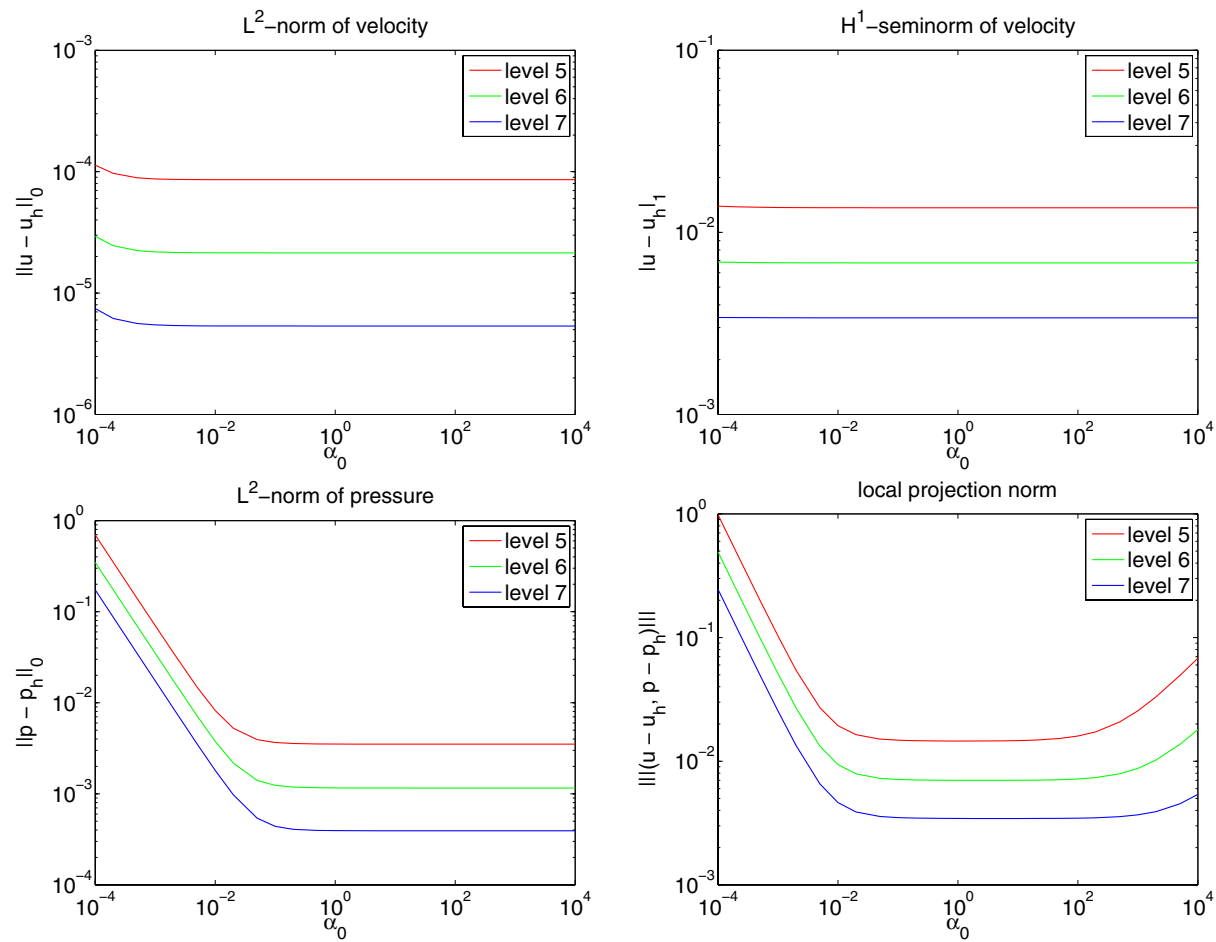

Figure 7. Dependence of norms on the parameter $\alpha_{0}$ for first order elements on triangles. 

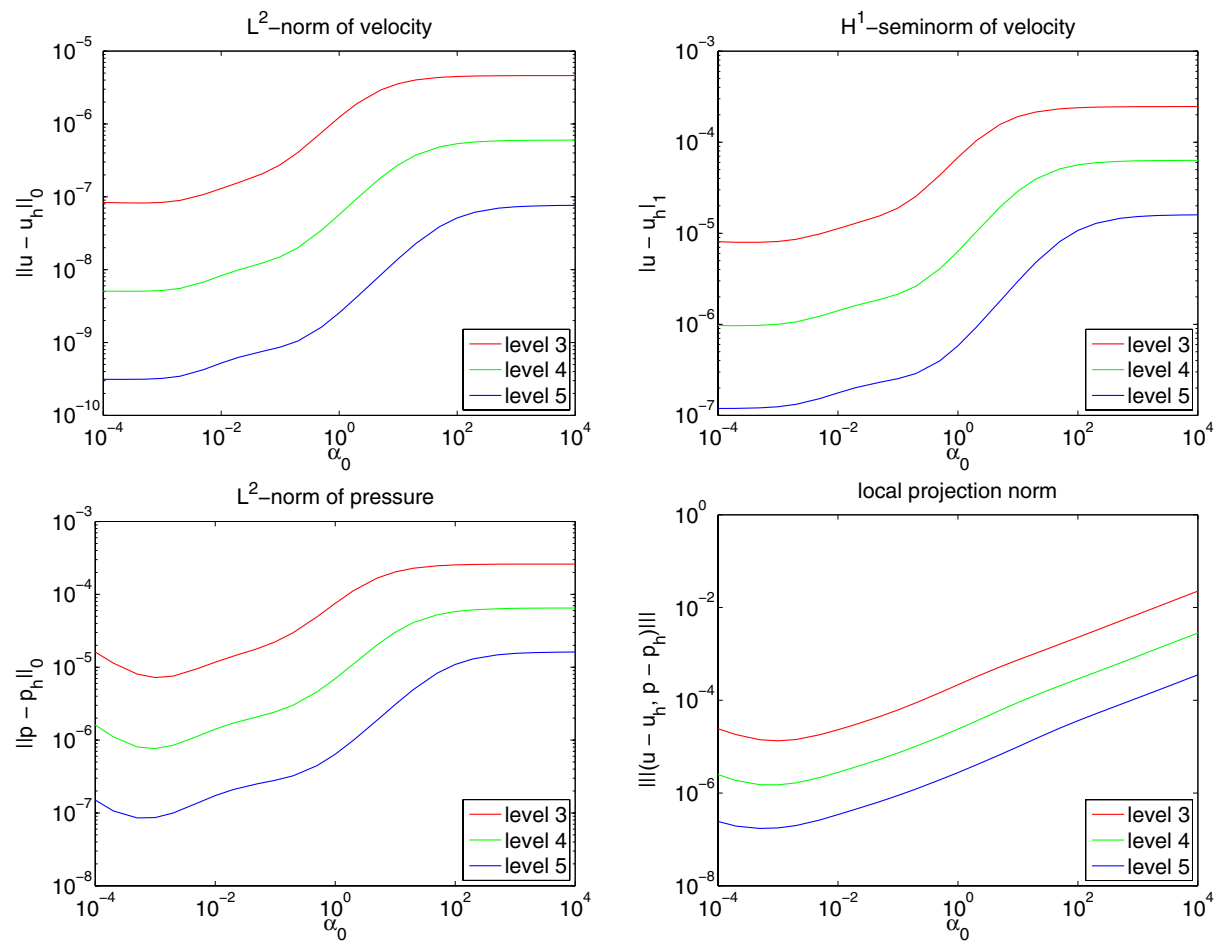

Figure 8. Dependence of norms on the parameter $\alpha_{0}$ for third order elements with difference 2 on quadrilaterals.

\section{ACKNOWLEDGMENT}

The authors would like to thank the German research foundation for partially supporting this research through Grant To143/9.

\section{REFERENCES}

1. T. Apel, Anisotropic finite elements. Local estimates and applications, Advances in Numerical Mathematics, Teubner, Leipzig, 1999. MR1716824 (2000k:65002)

2. D. N. Arnold, F. Brezzi, and M. Fortin, A stable finite element for the Stokes equation, Calcolo 21 (1984), 337-344. MR799997 (86m:65136)

3. W. Bai, The quadrilateral 'Mini' finite element for the Stokes problem, Comput. Methods Appl. Mech. Engrg. 143 (1997), 41-47. MR1442388 (98c:76061)

4. R. Becker and M. Braack, A finite element pressure gradient stabilization for the Stokes equations based on local projections, Calcolo 38 (2001), no. 4, 173-199. MR1890352 (2002m:65112)

5. _ A two-level stabilization scheme for the Navier-Stokes equations, Numerical mathematics and advanced applications (Berlin) (M. Feistauer, V. Dolejší, P. Knobloch, and K. Najzar, eds.), Springer-Verlag, 2004, pp. 123-130. MR.2121360 (2005k:65236)

6. P. Bochev and M. Gunzburger, An absolutely stable pressure-Poisson stabilized finite element method for the Stokes equation, SIAM J. Numer. Anal. 42 (2004), no. 3, 1189-1207. MR 2113682 (2005k:65223)

7. M. Braack and E. Burman, Local projection stabilization for the Oseen problem and its interpretation as a variational multiscale method, SIAM J. Numer. Anal. 43 (2006), no. 6, 2544-2566. MR2206447(2007a:65139) 
8. D. Braess and R. Sarazin, An efficient smoother for the Stokes problem, Appl. Numer. Math. 23 (1997), no. 1, 3-19. MR.1438078 (97k:65277)

9. F. Brezzi and M. Fortin, Mixed hybrid finite element methods, Springer-Verlag, 1991. MR.1115205 (92d:65187)

10. F. Brezzi and J. Pitkäranta, On the stabilization of finite element approximations of the Stokes problem, Efficient solutions of elliptic systems (W. Hackbusch, ed.), Notes on Numerical Fluid Mechanics, Vieweg, 1984, pp. 11-19. MR804083 (86j:65147)

11. P. G. Ciarlet, The finite element method for elliptic problems, SIAM, 2002. MR 1930132

12. P. Clément, Approximation by finite element functions using local regularization, RAIRO Anal. Numer. 9 (1975), 77-84. MR0400739 (53:4569)

13. R. Codina and J. Blasco, A finite element formulation for the Stokes problem allowing equal velocity-pressure interpolation, Comput. Methods Appl. Mech. Engrg. 143 (1997), 373-391. MR.1445157 (98j:76086)

14. J. Douglas and J. Wang, An absolutely stabilized finite element method for the Stokes problem, Math. Comp. 52 (1989), 495-508. MR958871 (89j:65069)

15. L. P. Franca and S. L. Frey, Stabilized finite element methods: II. The incompressible Navier-Stokes equations, Comput. Methods Appl. Mech. Engrg. 99 (1992), no. 2/3, 209233. MR1186727 (93i:76055)

16. L. P. Franca, S. P. Oliveira, and M. Sarkis, Continuous Q1/Q1 Stokes elements stabilized with non-conforming null edge average velocity functions, Math. Models Meth. Appl. Sci. (M3AS) 17 (2007), no. 3, 439-459. MR2311926

17. V. Girault and P.-A. Raviart, Finite Element Methods for Navier-Stokes Equation, Springer Series in Computational Mathematics, no. 5, Springer-Verlag, Berlin, 1986. MR851383 (88b:65129)

18. P. Hansbo and A. Szepessy, A velocity-pressure streamline diffusion method for the incompressible Navier-Stokes equations, Comput. Methods Appl. Mech. Engrg. 84 (1990), 175-192. MR:1087615 (91k:76109)

19. T. J. R. Hughes, L. P. Franca, and M. Balestra, A new finite element formulation for computational fluid dynamics. V: Circumventing the Babuška-Brezzi condition: A stable PetrovGalerkin formulation of the Stokes problem accomodating equal-order interpolations, Comput. Methods Appl. Mech. Engrg. 59 (1986), 85-99. MR868143 (89j:76015d)

20. V. John and G. Matthies, MooNMD-a program package based on mapped finite element methods, Comput. Vis. Sci. 6 (2004), no. 2-3, 163-169. MR2061275 (2005a:65132)

21. P. Knobloch and L. Tobiska, Stabilization methods of bubble type for the $Q_{1} / Q_{1}$-element applied to the incompressible Navier-Stokes equations, Math. Mod. Numer. Anal. (M2AN) 34 (2000), no. 1, 85-107. MR1735975 (2001d:65126)

22. G. Matthies, Mapped finite elements on hexahedra. Necessary and sufficient conditions for optimal interpolation errors, Numer. Algorithms 27 (2001), no. 4, 317-327. MR1880917 (2002j:65115)

23. G. Matthies, P. Skrzypacz, and L. Tobiska, A unified convergence analysis for local projection stabilisations applied to the Oseen problem, M2AN Math. Model. Numer. Anal. 41 (2007), no. $4,713-742$.

24. G. Matthies and L. Tobiska, The inf-sup condition for the mapped $Q_{k}-P_{k-1}^{\text {disc element in }}$ arbitrary space dimension, Computing 69 (2002), no. 2, 119-139. MR1954791 (2004g:65156)

25. P. Mons and G. Rogé, $L$ 'élément $Q_{1}$-bulle $/ Q_{1}$, Math. Mod. Numer. Anal. (M2AN) 26 (1992), 507-521. MR1163979 (93b:65174)

26. M. A. Olshanskii and A. Reusken, Grad-Div stabilization for the Stokes equations, Math. Comp. 73 (2004), 1699-1718. MR2059732 (2005a:65137)

27. F. Schieweck, Uniformly stable mixed hp-finite elements on multilevel adaptive grids with hanging nodes, Otto-von-Guericke-Universität Magdeburg, Preprint Nr. 2, 2007.

28. L. R. Scott and S. Zhang, Finite element interpolation of nonsmooth functions satisfying boundary conditions, Math. Comput. 54 (1990), no. 190, 483-493. MR1011446 (90j:65021)

29. L. Tobiska and G. Lube, A modified streamline diffusion method for solving the stationary Navier-Stokes equations, Numer. Math. 59 (1991), 13-29. MR.1103751 (92c:65141)

30. L. Tobiska and R. Verfürth, Analysis of a streamline diffusion finite element method for the Stokes and Navier-Stokes equations, SIAM J. Numer. Anal. 33 (1996), no. 1, 107-127. MR 1377246 (97e:65133) 
Institut für Analysis und Numerik, Otto-von-Guericke-Universität Magdeburg, Postfach 4120, D-39016 Magdeburg, Germany

E-mail address: ga.sashikumaar@mathematik.uni-magdeburg.de

FAKultät Für Mathematik, Ruhr-Universität Bochum, Universitätsstrasse 150 , D-44780 Bochum, Germany

E-mail address: gunar.matthies@ruhr-uni-bochum.de

Institut für Analysis und Numerik, Otto-von-Guericke-Universität Magdeburg, Postfach 4120, D-39016 Magdeburg, Germany

E-mail address: tobiska@mathematik.uni-magdeburg.de 